\title{
Doxorubicin Effect on Myocardial Metabolism as a Prerequisite for Subsequent Development of Cardiac Toxicity: A Translational ${ }^{18}$ F-FDG PET/CT Observation
}

\author{
Matteo Bauckneht ${ }^{1}$, Giulia Ferrarazzo ${ }^{1}$, Francesco Fiz ${ }^{1,2}$, Silvia Morbelli $^{1}$, Matteo Sarocchi ${ }^{3}$, Fabio Pastorino ${ }^{4}$, \\ Alberto Ghidella ${ }^{3}$, Elena Pomposelli ${ }^{1}$, Maurizio Miglino ${ }^{5}$, Pietro Ameri ${ }^{3}$, Laura Emionite ${ }^{6}$, Flavia Ticconi ${ }^{1}$, \\ Eleonora Arboscello ${ }^{7}$, Ambra Buschiazzo ${ }^{1}$, Elena Augusta Massimelli ${ }^{3}$, Salvatore Fiordoro ${ }^{1}$, Anna Borra ${ }^{1}$, \\ Vanessa Cossu ${ }^{1}$, Annalisa Bozzano ${ }^{1}$, Adalberto Ibatici ${ }^{5}$, Mirco Ponzoni ${ }^{4}$, Paolo Spallarossa ${ }^{3}$, Andrea Gallamini ${ }^{8}$, \\ Paolo Bruzzi ${ }^{9}$, Gianmario Sambuceti ${ }^{1}$, and Cecilia Marini ${ }^{10}$ \\ ${ }^{I}$ Nuclear Medicine, IRCCS-AOU San Martino-IST and University of Genoa, Genoa, Italy; ${ }^{2}$ Nuclear Medicine Unit, Department of \\ Radiology, Tübingen, Germany; ${ }^{3}$ Clinic of Cardiovascular Diseases, IRCCS-AOU San Martino-IST, Genoa, Italy; ${ }^{4}$ Unit of \\ Experimental Therapy in Oncology, IRCCS Gaslini, Genoa, Italy; ${ }^{5}$ Haematology Clinic, University of Genoa, IRCCS-AOU San \\ Martino-IST, Genoa, Italy; ${ }^{6}$ Animal Facility, IRCCS-AOU San Martino-IST, Genoa, Italy; ${ }^{7}$ Clinic of Internal Medicine 3, IRCCS-AOU \\ San Martino-IST, Genoa, Italy; ${ }^{8}$ Department of Research, Innovation, and Statistics, Lacassagne Cancer Centre, Nice, France; \\ ${ }^{9}$ Epidemiology Unit, IRCCS-AOU San Martino-IST, Genoa, Italy; and ${ }^{10}$ CNR Institute of Bioimaging and Molecular Physiology, \\ Section of Genoa, Milan, Italy
}

The present translational study aimed to verify whether serial ${ }^{18} \mathrm{~F}-$ FDG PET/CT predicts doxorubicin cardiotoxicity. Methods: Fifteen athymic mice were treated intravenously with saline $(n=5)$ or with 5 or $7.5 \mathrm{mg}$ of doxorubicin per kilogram ( $n=5$ each) and underwent dynamic small-animal PET beforehand and afterward to estimate left ventricular (LV) metabolic rate of glucose (MRGlu). Thereafter, we retrospectively identified 69 patients who had been successfully treated with a regimen of doxorubicin, bleomycin, vinblastine, and dacarbazine for Hodgkin disease (HD) and had undergone 4 consecutive ${ }^{18} \mathrm{~F}-\mathrm{FDG} \mathrm{PET} / \mathrm{CT}$ scans. Volumes of interest were drawn on LV myocardium to quantify mean SUV. All patients were subsequently interviewed by telephone (median follow-up, $30 \mathrm{mo}$ ); 36 of them agreed to undergo electrocardiography and transthoracic echocardiography. Results: In mice, LV MRGlu was $17.9 \pm 4.4 \mathrm{nmol} \times \mathrm{min}^{-1} \times$ $\mathrm{g}^{-1}$ at baseline. Doxorubicin selectively and dose-dependently increased this value in the standard-dose $\left(27.9 \pm 9 \mathrm{nmol} \times \min ^{-1} \times \mathrm{g}^{-1}, P<0.05\right.$ vs. controls) and high-dose subgroups $\left(37.2 \pm 7.8 \mathrm{nmol} \times \mathrm{min}^{-1} \times \mathrm{g}^{-1}\right.$, $P<0.01$ vs. controls, $P<0.05$ vs. standard-dose). In HD patients, LV SUV showed a progressive increase during doxorubicin treatment that persisted at follow-up. New-onset cardiac abnormalities appeared in 11 of 36 patients (31\%). In these subjects, pretherapy LV SUV was markedly lower with respect to the remaining patients $(1.53 \pm 0.9$ vs. $3.34 \pm 2.54$, respectively, $P<0.01)$. Multivariate analysis confirmed the predictive value of baseline LV SUV for subsequent cardiac abnormalities. Conclusion: Doxorubicin dosedependently increases LV MRGlu, particularly in the presence of low baseline ${ }^{18} \mathrm{~F}-\mathrm{FDG}$ uptake. These results imply that low myocardial ${ }^{18} \mathrm{~F}-\mathrm{FDG}$ uptake before the initiation of doxorubicin chemotherapy in HD patients may predict the development of chemotherapy-induced cardiotoxicity, suggesting that prospective clinical trials are warranted to test this hypothesis.

Received Jan. 31, 2017; revision accepted Apr. 18, 2017

For correspondence or reprints contact: Gianmario Sambuceti, Nuclear Medicine, IRCCS-AOU San Martino-IST and University of Genoa, Viale Benedetto XV, 6, Genoa 16132, Italy.

E-mail: sambuceti@unige.it.

Published online Jun. 23, 2017.

COPYRIGHT (c) 2017 by the Society of Nuclear Medicine and Molecular Imaging.
Key Words: doxorubicin; myocardial metabolism; FDG PET/CT

J Nucl Med 2017; 58:1638-1645

DOI: 10.2967/jnumed.117.191122

A

nthracycline cardiomyopathy represents a major drawback of chemotherapy for Hodgkin disease (HD) $(1,2)$. Although underlying mechanisms have not been fully elucidated, drug interference on the respiratory chain and consequent oxidative stress seem to play a major role $(2,3)$. This effect is eventually followed by an enhanced glucose consumption $(2,4)$ paralleled by an enhanced myocardial uptake of ${ }^{18} \mathrm{~F}-\mathrm{FDG}(5,6)$. However, the potential association between this index and anthracycline cardiotoxicity has been described in only one case report $(6)$, and its clinical potential remains uncertain.

The present translational study aimed to verify whether a response of cardiac ${ }^{18} \mathrm{~F}$-FDG uptake to doxorubicin might actually predict a late cardiotoxic effect. To this purpose, we first verified the dosedependent nature of doxorubicin action on myocardial metabolism by analyzing a series of cancer mouse models previously studied in our laboratory by small-animal PET scanning (7). Concurrently, we evaluated the serial PET/CT scans obtained from a cohort of HD patients to define the time sequence of doxorubicin metabolic effect and to verify its possible clinical correlates.

\section{MATERIALS AND METHODS}

\section{Animal Experiments}

Experiments were conducted under the Guide for the Care and Use of Laboratory Animals (8) and were approved by the local ethical committee. Animals were kept under the same dietary regimen and divided into 3 groups to be treated once a week for 3 wk with intravenous saline $(n=5)$ or either a $5 \mathrm{mg} / \mathrm{kg}$ (standard-dose, $n=5$ ) or $7.5 \mathrm{mg} / \mathrm{kg}$ (high-dose, $n=5$ ) dose of doxorubicin. Dynamic smallanimal PET imaging (Albira; Bruker US) was performed soon before and $6 \mathrm{~d}$ after chemotherapy. A Patlak graphical approach (9) was 
adopted to estimate left ventricular (LV) metabolic rate of glucose (MRGlu) and the corresponding index in skeletal muscle (SM) according to the standard procedure previously validated in our laboratory $(7,10)$. SUV was also estimated in LV myocardium and limb SM.

\section{Clinical Study}

Searching the keyword $H D$ in the database of all patients who underwent ${ }^{18}$ F-FDG PET/CT in our laboratory between January 2007 and December 2015, we identified 587 patients. The study population was thus selected according to the following inclusion criteria: no cardiovascular disease; no diabetes; normal baseline electrocardiogram and echocardiogram results; available staging ${ }^{18} \mathrm{~F}-\mathrm{FDG}$ PET/ CT scan (PET1); negative interim PET (PET2) results; completion of doxorubicin, bleomycin, vinblastine, and dacarbazine chemotherapy (doxorubicin dose, $40-50 \mathrm{mg} / \mathrm{m}^{2}$ per cycle); negative ${ }^{18} \mathrm{~F}-\mathrm{FDG}$ PET/CT results both at 4-6 wk after therapy (PET3) and at the 6-mo follow-up (PET4); and no subsequent HD relapse at late clinical follow-up. This process narrowed the final population down to 69 patients (Supplemental Fig. 1; supplemental materials are available at http://jnmt. snmjournals.org). The myocardial metabolic pattern in these patients was compared with the corresponding findings in 69 sex- and agematched subjects selected from our database (11). The institutional review board approved this study, and all subjects gave written informed consent to the imaging procedure, as part of our routine clinical care.

The ${ }^{18} \mathrm{~F}$-FDG intravenous injection was preceded by a minimum of $6 \mathrm{~h}$ of fasting and serum glucose level control. All ${ }^{18} \mathrm{~F}$ FDG PET/CT scans were acquired according to the conventional procedure, using a Hirez16 PET/CT hybrid system (Siemens Medical Solutions). Two volumes of interest were manually drawn on the LV myocardium and on the SM (longissimus thoracis) to estimate the LV SUV and SM SUV, respectively. CT images were used to identify the myocardium when there was no cardiac uptake.

All 69 patients were interviewed by telephone; 36 of them agreed to undergo a clinical reevaluation (14 women and 22 men; mean age, $39 \pm 14$ y; age range, 21-68 y). An experienced cardiologist, unaware of the PET findings, confirmed the absence of interval development of palpitations, syncope, chest pain, or dyspnea and complemented the physical examination with electrocardiography and with echocardiographic evaluation of wall thicknesses, LV diameters, ejection fraction, and diastolic function by E/A wave ratio and $\mathrm{E}$ wave deceleration time (12).

\section{Statistical Analysis}

All data are presented as mean $\pm \mathrm{SD}$ or proportions. Differences between paired and unpaired continuous data were analyzed by the Student $t$ test, as appropriate. Categoric variables were analyzed using the $\chi^{2}$ test. A probability value of less than 0.05 was considered statistically significant. In instances of a skewed data distribution, values were transformed using a natural logarithmic transform. Finally, the ability of LV SUV1 to predict the occurrence of cardiotoxicity while adjusting for various potential confounders was tested by multivariate logistic regression analysis. The presence or absence of cardiac abnormalities was tested with respect to the following baseline covariates: age, sex, Ann Arbor stage, mediastinal irradiation, cumulative administered doxorubicin dose, and baseline myocardial ${ }^{18} \mathrm{~F}-\mathrm{FDG}$ uptake. Since clinical assessment for the presence of cardiac abnormalities occurred at various times (from 8 to $26 \mathrm{mo}$ ) after the administration of doxorubicin, bleomycin, vinblastine, and dacarbazine, follow-up duration was also included in the model as a covariate. Because of collinearity between Ann Arbor staging and mediastinal irradiation, these two variables could not be included in the same model. Their role as confounders was tested separately with all the other covariates included. Because the

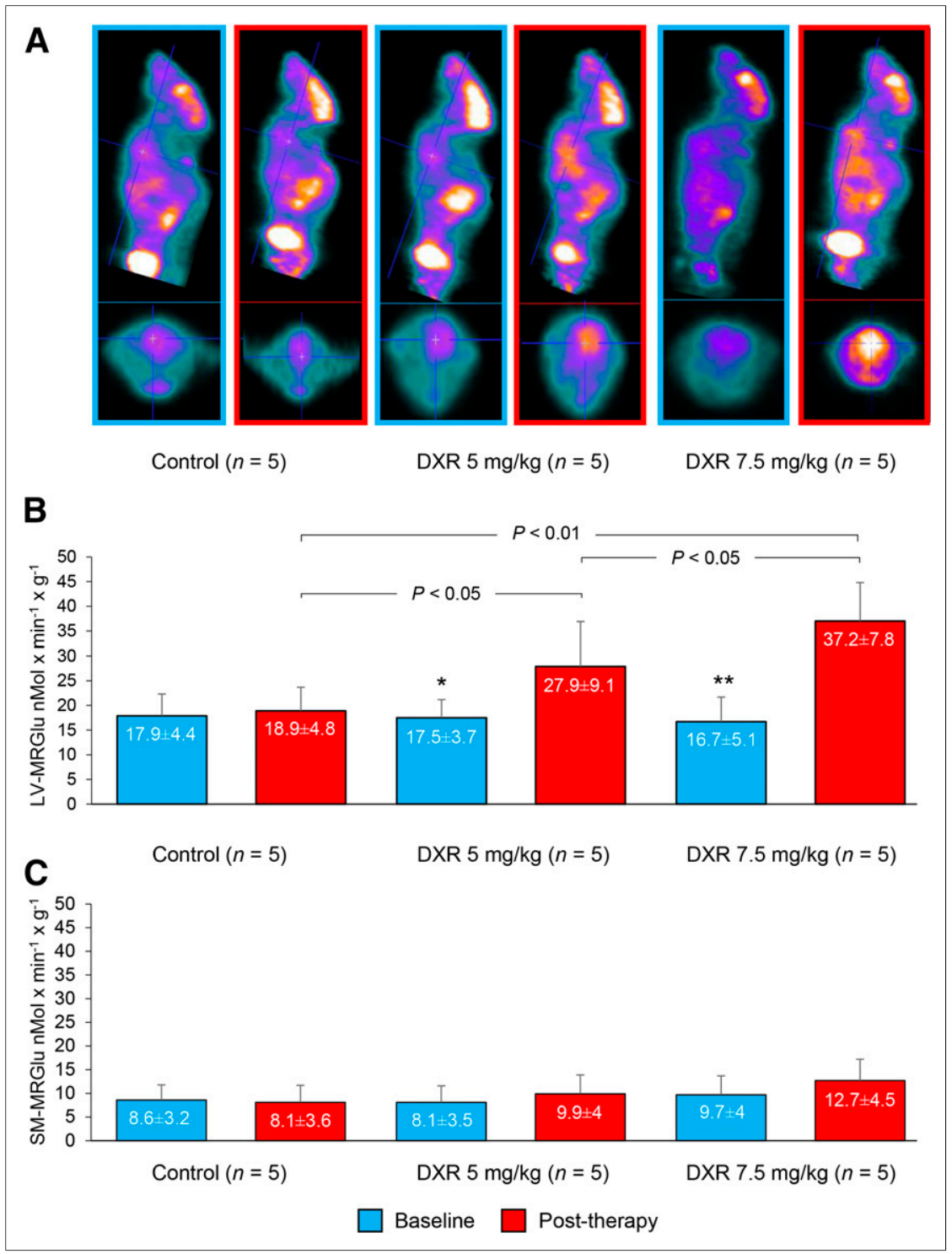

FIGURE 1. Dose-dependent doxorubicin effect on LV MRGlu. (A) Axial and sagittal planes of small-animal PET studies on mice before and after treatment with saline or with 5 or $7.5 \mathrm{mg} / \mathrm{kg}$ dose of doxorubicin. (B) Doxorubicin administration was followed by significant increase in LV MRGlu, as opposed to stable values in untreated mice. Moreover, dose-dependent nature of doxorubicin metabolic effect was confirmed by significant difference between posttherapy scans in animals treated with standard or high doses. (C) Doxorubicin administration did not affect SM MRGlu. ${ }^{*} P<0.05$ vs. after treatment. ${ }^{* \star} P<0.01$ vs. after treatment. DXR $=$ doxorubicin. 
results of the two analyses were similar, only those of the first one are reported. The multivariate analyses proceeded by means of a backward stepwise procedure, based on the likelihood ratio test, with a $P$ value of at least 0.1 for removal. The estimated coefficients with their standard errors were used to compute the odds ratios with $95 \%$ confidence intervals. The same model was also fitted separately for each covariate to estimate the univariate odds ratios with $95 \%$ confidence intervals. Statistical analyses were performed using a dedicated software application, SPSS (version 21.0, IBM).

\section{RESULTS}

\section{In Vivo Animal Experiments}

In animal models, doxorubicin increased cardiac glucose disposal (Fig. 1A) without affecting either body weight or serum glucose level (data not shown). At compartmental analysis of dynamic PET scans (Fig. 1B), LV MRGlu remained stable in control mice (from $17.9 \pm 4.4$ to $18.9 \pm 4.8 \mathrm{nmol} \times \min ^{-1} \times \mathrm{g}^{-1}$, $P=$ not statistically significant). Standard dose increased LV MRGlu from $17.5 \pm 3.7$ to $27.9 \pm 9.1 \mathrm{nmol} \times \min ^{-1} \times \mathrm{g}^{-1}(P<0.05$ vs. controls; $P<0.05$ vs. corresponding baseline). This effect was significantly more evident at the high drug dose, which augmented LV
MRGlu from $16.7 \pm 5.1$ to $37.2 \pm 7.8 \mathrm{nmol} \times \min ^{-1} \times \mathrm{g}^{-1}(P<$ 0.01 vs. controls and corresponding baseline; $P<0.05$ vs. standard dose). As expected, the analysis of LV SUV strictly reproduced all these findings (Supplemental Figs. 2A and 2B).

Cardiac selectivity of doxorubicin metabolic effect was confirmed by the lack of SM response in all groups. In fact, both SM MRGlu (Fig. 1C) and SM SUV (Supplemental Fig. 2B) remained remarkably stable before and after treatment. Consequently, the ratio LV SUV/SM SUV remained stable at baseline values in sham mice (3.28 \pm 0.9$)$ but increased in a dose-dependent fashion after chemotherapy (to $4.42 \pm 1.02$ and to $6.43 \pm 1.96$ in standard and high doxorubicin doses $[P<0.05$ and $P<0.01]$, respectively).

\section{Overall Doxorubicin Effect on Cardiac Metabolism in Humans}

Clinical data and time intervals between the 4 PET/CT studies of the patient population and the controls are reported in Table 1. Baseline LV SUV was similarly distributed in the controls, in the 69 studied subjects, and in the 35 excluded patients because of positive PET2 results (Fig. 2A). Similarly, no difference in age, sex, or Ann Arbor stage was observed between the 69 recruited patients and the 138 excluded ones because of either positive

TABLE 1

Baseline Clinical Characteristics of HD and Control Enrolled Patients

\begin{tabular}{|c|c|c|c|}
\hline Characteristic & HD & Control & $P$ \\
\hline Age (y) & $39 \pm 13$ (range, $19-58)$ & $41 \pm 8$ (range, $20-72$ ) & NS \\
\hline Male sex & $37 / 69(53 \%)$ & $35 / 69(50 \%)$ & NS \\
\hline Weight (kg) & $67.1 \pm 12$ & $76.5 \pm 7$ & $<0.05$ \\
\hline Glycemia at ${ }^{18} \mathrm{~F}-\mathrm{FDG}$ injection (mg/dL) & $79 \pm 7$ (range, 61-101) & $83 \pm 11$ (range, 62-94) & NS \\
\hline \multicolumn{4}{|l|}{ Cardiovascular risk profile } \\
\hline Hypertension & $6 / 69(8 \%)$ & $15 / 69(21 \%)$ & $<0.01$ \\
\hline Tobacco use & $19 / 69(27 \%)$ & $30 / 69(43 \%)$ & $<0.05$ \\
\hline Total cholesterol & $183.7 \pm 30$ & $188 \pm 53$ & NS \\
\hline Low-density lipoprotein & $114.5 \pm 32$ & $120 \pm 25$ & NS \\
\hline Triglycerides & $121.3 \pm 49$ & $129.7 \pm 57$ & NS \\
\hline Creatinine & $0.8 \pm 0.1$ & $0.85 \pm 0.2$ & NS \\
\hline Family history of CAD & 7/69 (10\%) & $5 / 69(7 \%)$ & NS \\
\hline \multicolumn{4}{|l|}{ Time intervals between PET studies } \\
\hline PET1-PET2 (d) & $73.7 \pm 21$ & $99 \pm 90$ & $<0.05$ \\
\hline PET2-PET3 (d) & $148 \pm 70$ & $167 \pm 98$ & NS \\
\hline PET3-PET4 (d) & $195 \pm 92$ & $229 \pm 100$ & NS \\
\hline Overall PET1-PET4 (d) & $427 \pm 198$ & $448 \pm 141$ & NS \\
\hline \multicolumn{4}{|l|}{ Baseline Ann-Arbor staging } \\
\hline I stage & 7/69 (10\%) & - & - \\
\hline II stage & $42 / 69(60 \%)$ & - & - \\
\hline III stage & $8 / 69(12 \%)$ & - & - \\
\hline IV stage & $12 / 69(17 \%)$ & - & - \\
\hline B symptoms & $10 / 69(14 \%)$ & - & - \\
\hline Mediastinal radiotherapy & $35 / 69$ (55\%) & - & - \\
\hline Total administered doxorubicin dose (mg) & $456.6 \pm 103$ & - & - \\
\hline
\end{tabular}

NS = not statistically significant; CAD = coronary artery disease.

Qualitative data are expressed as numbers followed by percentages in parentheses; continuous data are expressed as mean \pm SD. 


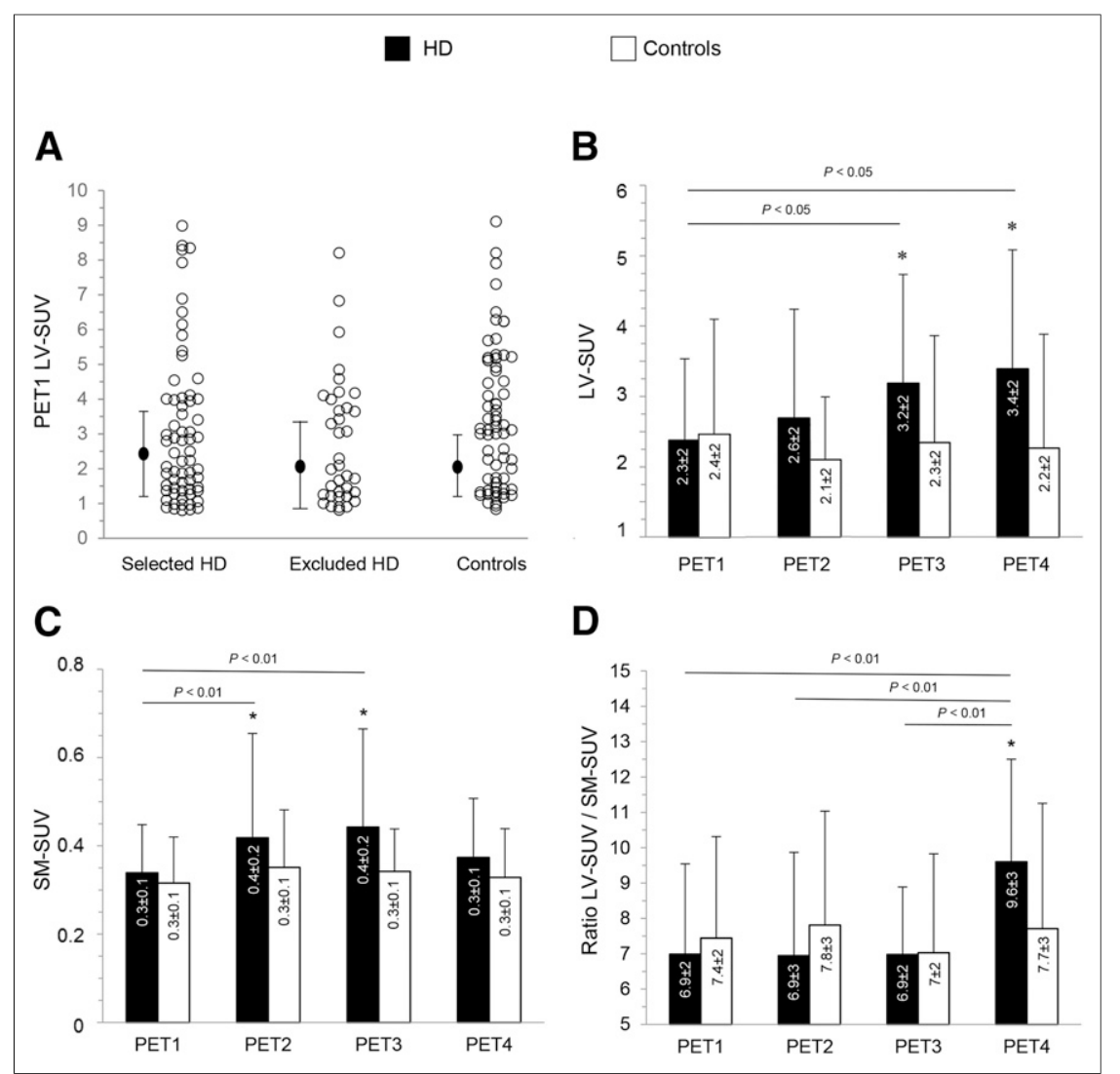

FIGURE 2. Myocardial and SM divergent metabolic pattern in HD population. (A) Distribution of baseline LV SUV was similar between 69 enrolled subjects, excluded patients, and controls. (B) In HD patients, LV SUV progressively increased from PET1 to PET3, remaining relatively stable at PET4. By contrast, it remained unchanged in controls. (C) Divergent nature of SM doxorubicin effect can be appreciable at PET4. (D) LV SUV/SM SUV ratio at the 4 time points. ${ }^{*} P<0.05$ vs. control.

PET2 results $(n=35)$ or other reasons $(n=103)$ (Supplemental Fig. 1; Supplemental Table 1), suggesting a low likelihood of selection bias.

In the HD population, LV SUV was $2.37 \pm 1.6$ at baseline, showed a progressive increase during doxorubicin treatment up to PET3, and remained persistently elevated at PET4 (Fig. 2B). By contrast, overall cardiac uptake remained unchanged in control subjects throughout the study period. Accordingly, HD patients showed significantly higher LV SUVs with respect to controls both at PET3 and at PET4 (Fig. 2B).

Again, the selective nature of cardiac response was confirmed by the divergent behavior of SM metabolism, which was only scarcely and transiently affected by doxorubicin (Fig. 2C). Therefore, the ratio between LV and SM SUV remained stable during treatment, whereas it significantly increased during follow-up (Fig. 2D) to values significantly higher than those observed in control subjects.

Despite this trend in average values, patients' response to chemotherapy only partially reproduced the repeatability of doxorubicin action on myocardial metabolism observed in animal experiments. Although no patient showed a progressive reduction in myocardial tracer retention during or after therapy, doxorubicin effect on ${ }^{18} \mathrm{~F}$ FDG myocardial uptake was heterogeneous and largely independent of the cumulative drug dose (Supplemental Fig. 3).

\section{Myocardial Function Assessment}

During the interval between PET4 and the cardiologic interview (median time from PET4, $30 \mathrm{mo}$; range, 3-96 mo), none of the 69 patients reported any hospitalization potentially related to cardiac disorders. In the 36 patients who agreed to undergo a clinical evaluation, the median time from treatment start to visit was 27 mo (range, 8-96 mo). Electrocardiography or echocardiography documented new-onset abnormalities in 11 patients $(31 \%)$ ( 4 women and 7 men; mean age, $44 \pm 17 \mathrm{y}$; age range, 21-66 y; Table 2). Signs of possible cardiotoxicity were dyspnea associated with a decrease in LV ejection fraction $(n=2)$, atrial fibrillation $(n=1)$, the appearance of negative T waves in the anterior leads $(n=2)$, and an alteration in the diastolic mitral flow profile (inversion of E/A wave ratio) at Doppler examination $(n=6)$.

According to the inclusion criteria, pretherapy LV dimensions and function were normal and were remarkably similar between the two subgroups. By contrast, average LV ejection fraction significantly decreased after doxorubicin in the 11 patients with cardiotoxic response and became significantly lower with respect to the remaining 25 patients (Table 2; Fig. 3).

The appearance of cardiac abnormality was not related to differences in sex, age, mediastinal radiotherapy, total drug dose, or follow-up duration (Table 2). By contrast, it was associated with markedly lower LV SUVs at baseline with respect to both the remaining 25 patients $(1.53 \pm$ 0.9 vs. $3.34 \pm 2.54$, respectively, $P<0.01$, Figs. 4A and $4 \mathrm{~B}$ ) and the control subjects (3.2 $\pm 1.7, P<0.01$ vs. abnormal and $P=$ not statistically significant vs. negative clinical follow-up). This difference tended to progressively disappear over time (Fig. 4C). In fact, ${ }^{18} \mathrm{~F}-\mathrm{FDG}$ uptake significantly and progressively increased in the 11 patients with late cardiac abnormalities (Fig. 4D). This trend persisted even after doxorubicin discontinuation, as opposed to the remaining 25 subjects with negative follow-up, in whom the cardiac metabolic pattern remained relatively stable during and after doxorubicin, bleomycin, vinblastine, and dacarbazine (Fig. 4E). Multivariate analysis confirmed that baseline ${ }^{18} \mathrm{~F}-\mathrm{FDG}$ uptake was strongly associated with the subsequent development of cardiac abnormalities, providing additive predictive power with respect to conventional risk stratification (Table 3).

\section{DISCUSSION}

The present study documented that doxorubicin influences myocardial glucose consumption. The dose-dependent nature of this metabolic effect was well evident and reproducible in animal experiments, whereas it was largely heterogeneous in patients according to baseline metabolic pattern. Relatively preserved cardiac ${ }^{18}$ F-FDG retention predicted a virtual absence of response of cardiac metabolism to doxorubicin. By contrast, patients with low baseline tracer uptake displayed a systematic metabolic reaction to doxorubicin: myocardial SUV progressively increased during the therapy; this state of increased glucose consumption persisted for 
TABLE 2

Demographic and Clinical Data of HD Patients with Normal Follow-up Findings with Respect to Those with Abnormal Follow-up Findings

\begin{tabular}{|c|c|c|c|}
\hline Characteristic & Normal follow-up $(n=25)$ & Abnormal follow-up $(n=11)$ & $P$ \\
\hline Age (y) & $36.8 \pm 12$ & $44.5 \pm 17$ & NS \\
\hline Male sex & $14(56 \%)$ & 7 (63\%) & NS \\
\hline Weight (kg) & $68.9 \pm 13$ & $70.7 \pm 12$ & NS \\
\hline Glycemia at PET1 (mg/dL) & $79.2 \pm 5$ & $82.4 \pm 4$ & NS \\
\hline \multicolumn{4}{|l|}{ Cardiovascular risk profile } \\
\hline Hypertension & $2(8 \%)$ & $1(9 \%)$ & NS \\
\hline Tobacco use & $5(20 \%)$ & $2(18 \%)$ & NS \\
\hline Total cholesterol & $186 \pm 28$ & $181 \pm 33$ & NS \\
\hline Low-density lipoprotein & $119.6 \pm 28$ & $113.3 \pm 31$ & NS \\
\hline Triglycerides & $117.2 \pm 56$ & $122.9 \pm 49$ & NS \\
\hline Creatinine & $0.8 \pm 0.1$ & $0.7 \pm 0.1$ & NS \\
\hline Family history of CAD & $2(8 \%)$ & $1(9 \%)$ & NS \\
\hline \multicolumn{4}{|l|}{ Baseline Ann Arbor staging } \\
\hline I stage & $3(12 \%)$ & $2(18 \%)$ & NS \\
\hline II stage & $15(60 \%)$ & $5(45 \%)$ & NS \\
\hline III stage & $3(12 \%)$ & $2(18 \%)$ & NS \\
\hline IV stage & $4(16 \%)$ & $2(18 \%)$ & NS \\
\hline B symptoms & $5(20 \%)$ & $2(18 \%)$ & NS \\
\hline Mediastinal radiotherapy & $13(52 \%)$ & $5(45 \%)$ & NS \\
\hline Total administered doxorubicin dose (mg) & $430.9 \pm 109$ & $421.3 \pm 107$ & NS \\
\hline Clinical follow-up duration (d) & $1,121 \pm 874$ & $860 \pm 665$ & NS \\
\hline \multicolumn{4}{|l|}{ Follow-up clinical data } \\
\hline Chest pain & - & - & - \\
\hline Dyspnea & - & $2(18 \%)$ & - \\
\hline Syncope & - & - & - \\
\hline Palpitations & - & $2(18 \%)$ & - \\
\hline Follow-up ECG abnormalities & - & $3(27 \%)^{\star}$ & - \\
\hline \multicolumn{4}{|l|}{ Baseline echocardiography } \\
\hline End-diastolic diameter (mm) & $49.1 \pm 2$ & $48 \pm 3$ & NS \\
\hline End-systolic diameter (mm) & $29 \pm 3$ & $31 \pm 2$ & NS \\
\hline Fractional shortening (\%) & $44 \% \pm 4 \%$ & $46 \% \pm 3 \%$ & NS \\
\hline LV ejection fraction & $59.8 \pm 2.1$ & $59.3 \pm 1.7$ & NS \\
\hline Diastolic dysfunction & $0(0 \%)$ & $0(0 \%)$ & NS \\
\hline \multicolumn{4}{|l|}{ Follow-up echocardiography } \\
\hline End-diastolic diameter (mm) & $49.2 \pm 1$ & $49.3 \pm 5$ & NS \\
\hline End-systolic diameter (mm) & $27.2 \pm 3$ & $33.6 \pm 11$ & NS \\
\hline Fractional shortening (\%) & $44 \% \pm 4 \%$ & $38 \% \pm 5 \%{ }^{\dagger}$ & $<0.05$ \\
\hline LV ejection fraction & $60.3 \pm 2$ & $55 \pm 7^{\dagger}$ & $<0.05$ \\
\hline Diastolic dysfunction & $0(0 \%)$ & $5(45 \%)$ & $<0.01$ \\
\hline
\end{tabular}

*1 patient developed atrial fibrillation; 2 patients developed negative T waves in anterior leads.

${ }^{\dagger} P<0.05$ with respect to corresponding baseline.

$\mathrm{NS}=$ not statistically significant; ECG = electrocardiographic; CAD = coronary artery disease.

Qualitative data are expressed as numbers followed by percentages in parentheses; continuous data are expressed as mean \pm SD.

1642 The Journal of Nuclear Medicine • Vol. 58 • No. 10 - October 2017 


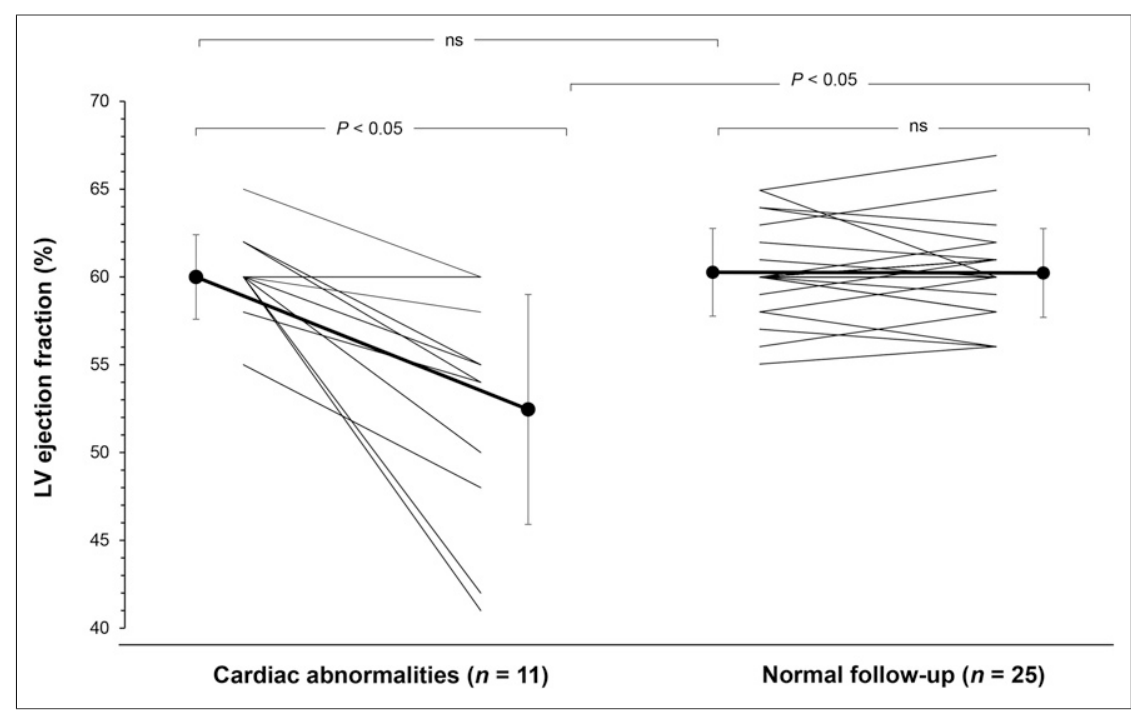

FIGURE 3. Individual and average values of LV ejection fraction in 2 subgroups of patients with normal and abnormal cardiac follow-up. ns = not statistically significant. followed by a late decrease in ${ }^{18} \mathrm{~F}-\mathrm{FDG}$ uptake, at least in rats treated with doxorubicin doses lower than that used in the present study (19).

Besides energy metabolism, the evident doxorubicin capability of inducing myocardial lipid peroxidation may contribute to increased glucose intake since glutathione oxidation inevitably accelerates glucose flux through the pentose phosphate pathway. This hypothesis is in accordance with the recent indication that endoplasmic reticulum oxidative stress is a major determinant of doxorubicin cardiotoxicity (20). Similarly, recent evidence by our laboratory suggests that ${ }^{18} \mathrm{~F}$-FDG uptake in cancer cells is relatively independent of overall glucose consumption, selectively tracking a peculiar pathway located within the endoplasmic reticulum dedicated to preserving redox equilibrium (10). months after treatment. This metabolic pattern correlated with the onset of late electrocardiographic or echocardiographic abnormalities. Multivariate analysis confirmed the low myocardial ${ }^{18} \mathrm{~F}-\mathrm{FDG}$ uptake before therapy as the most potent predictor of subsequent cardiac alterations, whose power even overcame the predictive value of acknowledged risk factors such as mediastinal irradiation or total doxorubicin dose (2).

\section{Doxorubicin Effect on Cardiac Metabolism}

The analysis of doxorubicin effect on myocardial ${ }^{18} \mathrm{~F}-\mathrm{FDG}$ uptake has been hampered by the large variability of myocardial metabolic pattern under fasting conditions (13), commonly attributed to the dietary regimen in the days before ${ }^{18} \mathrm{~F}-\mathrm{FDG}$ PET/CT (14). The experimental part of our study ruled out this confounding interference to document that doxorubicin dose-dependently and rapidly increased myocardial SUV in mice fed a standardized diet. No data about cardiac function were available in these animals, and thus the present study did not elucidate whether the increased ${ }^{18} \mathrm{~F}$-FDG uptake was a marker of an early contractile impairment. Nevertheless, this effect occurred in all animals and was induced by the same drug doses that could trigger the late appearance of both electrocardiographic abnormalities and contractile impairment (15). Along this line, it is conceivable that the observed metabolic response might represent an early marker of cardiac toxicity at least partially able to predict the development of clinically evident anthracycline-related cardiac dysfunction.

Unfortunately, the retrospective nature of data selection prevented any possible evaluation of the mechanisms underlying the accelerated glucose consumption. However, Hrelia et al. previously reported that $1 \mu \mathrm{M}$ doxorubicin almost doubles the uptake of the ${ }^{18} \mathrm{~F}$-FDG analog 2-deoxyglucose, enhancing the expression of glucose transporter 1 in neonatal rat ventricular cardiomyocytes (16). This response might reflect the acknowledged capability of doxorubicin to inhibit fatty acid oxidation and mitochondrial function (17), thus increasing glucose intake via the phosphorylation of adenosine monophosphate-activated protein kinase (18). However, the direct relationship between this metabolic shift and energy depletion remains largely elusive, mostly because the increase in glucose consumption has been found to be transient (16) and

\section{Doxorubicin Effect on HD Patients}

In the clinical setting, several confounding factors hamper a precise definition of the link between trends in ${ }^{18} \mathrm{~F}-\mathrm{FDG}$ uptake and doxorubicin cardiotoxic effects. On one hand, obvious ethical considerations prevented an accurate definition of dose dependency of this drug action. On the other hand, changes in cardiac energy expenditure (e.g., changes in LV rate-pressure product) were not assessed as part of the clinical imaging procedure and therefore it is uncertain if a portion of the observed increases in LV SUVs might have reflected changes in myocardial workload. However, no patient complained of symptoms suggesting a progressive increase in heart rate or arterial pressure to levels able to induce a measurable increase in LV MRGlu during treatment and after its discontinuation (21). Similarly, follow-up cardiologic examination did not report tachycardia or new-onset hypertension in any of the 36 tested patients, suggesting that changes in workload are unlikely to have a major impact on the observed cardiac metabolic responses to doxorubicin. Finally, we evaluated only patients with negative interim PET results to avoid the interference of changes in chemotherapy regimen. Accordingly, negative interim PET predicts the highest prevalence of disease remission and thus identifies patients in whom doxorubicin cardiotoxicity is a major drawback. This decision might have introduced possible selection biases. However, the clinical picture and LV SUV were similarly distributed in the enrolled population, in the excluded HD patients, and in the control subjects.

Together with its systematic nature, the prolonged duration of cardiac ${ }^{18} \mathrm{~F}$-FDG response to doxorubicin intrinsically implies that the known variability of myocardial ${ }^{18}$ F-FDG uptake cannot be attributed simply to the dietary regimen on the days immediately preceding the PET study (14). Rather, it identifies a direct and selective doxorubicin action on the myocardium. In fact, the increase in cardiac ${ }^{18} \mathrm{~F}$-FDG uptake persisted for 6 mo after doxorubicin, bleomycin, vinblastine, and dacarbazine discontinuation, when SM had turned back to a baseline metabolic pattern. The different time trend documented in our patient cohort nicely reproduces the different time course in respiratory impairment in the two tissues (22) and the selective nature of mitochondrial damage induced by doxorubicin in the myocardium (23) of experimental models. 




FIGURE 4. (A and B) Divergent myocardial metabolic pattern between example HD patients with abnormal (A) and normal (B) cardiac followup. (C) ${ }^{18} \mathrm{~F}-\mathrm{FDG}$ uptake on 4 PET/CT scans in 2 subgroups shows that baseline LV SUV was markedly lower in patients with late cardiac abnormalities than in remaining 25 patients $\left({ }^{*} P<0.01\right)$. (D) ${ }^{18} \mathrm{~F}-\mathrm{FDG}$ uptake significantly and progressively increased in 11 patients with late cardiac abnormalities. (E) This trend persisted even after doxorubicin discontinuation, as opposed to remaining 25 subjects.
Differently from PET data, cardiac evaluation was available in only 36 of the 69 selected HD patients. This limitation was obviously caused by the retrospective nature of our analysis and implies that the only information available for the remaining 33 patients is the absence of hospital admissions. Similarly, only 2 patients developed symptomatic LV dysfunction and only 1 patient atrial fibrillation. However, the applied criteria identified a decrease in LV ejection fraction that, though modest, is an acknowledged predictor of subsequent cardiotoxic evolution (24). Unfortunately, the present data do not elucidate whether baseline ${ }^{18}$ F-FDG uptake predicts a progression of contractile impairment in high-risk HD patients with previous cardiovascular diseases. This task would have required a multicenter approach and large patient sample to account for the inevitable interference of heterogeneities in the progression rate of preexisting cardiac disorders and in the necessary adaptation of chemotherapy schemes.

\section{CONCLUSION}

The present study highlighted a dose-dependent action of doxorubicin on myocardial metabolism. In the clinical setting, this response seems to be present only (or mainly) in the subset of patients in whom a low baseline myocardial ${ }^{18} \mathrm{~F}$-FDG uptake predicted a progressive increase in cardiac glucose consumption during and after chemotherapy, as well as a higher incidence of cardiac abnormalities.

The potential impact of these findings and their correct introduction into clinical practice require further prospective studies. Nevertheless, their relevance relies on the observation of a predictive value for cardiac ${ }^{18}$ F-FDG uptake in the PET sessions performed, under fasting conditions, for staging purposes. Should these data be confirmed, the selection of candidate alternative therapeutic protocols or of candidate cardioprotective treatments might be possible without the need for a further scan by adopting specific procedures to optimize the study of myocardial metabolism.

\section{DISCLOSURE}

Funding was provided by the Italian Association for Cancer Research (IG 14231 and IG 18474 to Mirco Ponzoni; IG 15426 to Cecilia Marini). The study was also partially supported by the program "Ricerca Corrente," line "Guest-Cancer Interactions," by Compagnia di San Paolo (project ID Prot.: 2015.AAI4110. U4917). Fabio Pastorino is supported by Fondazione Umberto

TABLE 3

Prediction of Cardiac Abnormalities: Uni- and Multivariate Analysis

\begin{tabular}{|c|c|c|c|c|c|c|c|}
\hline \multirow[b]{2}{*}{ Characteristic } & \multirow[b]{2}{*}{ df } & \multicolumn{3}{|c|}{ Univariate analysis } & \multicolumn{3}{|c|}{ Multivariate analysis } \\
\hline & & $P$ & OR & $95 \% \mathrm{Cl}$ & $P$ & OR & $95 \% \mathrm{Cl}$ \\
\hline Sex & 1 & 0.760 & 0.8 & $0.19-3.35$ & 0.142 & * & \\
\hline Age (y) & 1 & 0.140 & 1.04 & $0.99-1.09$ & 0.339 & * & \\
\hline Anne Arbor stage & 1 & 0.882 & 0.94 & $0.41-2.12$ & 0.694 & * & \\
\hline Mediastinal radiotherapy & 1 & 0.718 & 1.30 & $0.31-5.39$ & 0.711 & * & \\
\hline Cumulative doxorubicin dose (mg) & 1 & 0.963 & 1.00 & $0.99-1.01$ & 0.486 & * & \\
\hline Baseline LV SUV & 1 & 0.030 & 0.18 & $0.03-0.85$ & $<0.001$ & 0.065 & $0.006-0.74$ \\
\hline Follow-up time (d) & 1 & 0.364 & 1.00 & $0.99-1.001$ & 0.408 & * & \\
\hline
\end{tabular}

${ }^{\star}$ Excluded from final model.

$\mathrm{df}=$ degrees of freedom; OR $=$ odds ratio; $\mathrm{Cl}=$ confidence limit. 
Veronesi and Istituto G. Gaslini Awards. No other potential conflict of interest relevant to this article was reported.

\section{REFERENCES}

1. Jemal A, Ward E, Hao Y, Thun M. Trends in the leading causes of death in the United States, 1970-2002. JAMA. 2005;294:1255-1259.

2. Takemura G, Fujiwara H. Doxorubicin-induced cardiomyopathy from the cardiotoxic mechanisms to management. Prog Cardiovasc Dis. 2007;49:330-352.

3. Sarvazyan N. Visualization of doxorubicin-induced oxidative stress in isolated cardiac myocytes. Am J Physiol. 1996;271:H2079-H2085.

4. Israel O, Weiler-Sagie M, Rispler S, et al. PET/CT quantitation of the effect of patient-related factors on cardiac ${ }^{18}$ F-FDG uptake. J Nucl Med. 2007;48:234239.

5. Borde C, Kand P, Basu S. Enhanced myocardial fluorodeoxyglucose uptake following Adriamycin-based therapy: evidence of early chemotherapeutic cardiotoxicity? World J Radiol. 2012;4:220-223.

6. Gorla AK, Sood A, Prakash G, Parmar M, Mittal BR. Substantial increase in myocardial FDG uptake on interim PET/CT may be an early sign of Adriamycininduced cardiotoxicity. Clin Nucl Med. 2016;41:462-463.

7. Cossu I, Bottoni G, Loi M, et al. Neuroblastoma-targeted nanocarriers improve drug delivery and penetration, delay tumor growth and abrogate metastatic diffusion. Biomaterials. 2015;68:89-99.

8. Guide for the Care and Use of Laboratory Animals. Bethesda, MD: National Institutes of Health; 1985. NIH publication 85-23.

9. Patlak CS, Blasberg RG, Fenstermacher JD. Graphical evaluation of blood-to brain transfer constants from multiple-time uptake data. J Cereb Blood Flow Metab. 1983;3:1-7.

10. Marini C, Ravera S, Buschiazzo A, et al. Discovery of a novel glucose metabolism in cancer: the role of endoplasmic reticulum beyond glycolysis and pentose phosphate shunt. Sci Rep. 2016;6:25092.

11. Sambuceti G, Brignone M, Marini C, et al. Estimating the whole bone-marrow asset in humans by a computational approach to integrated PET/CT imaging. Eur J Nucl Med Mol Imaging. 2012;39:1326-1338.

12. Gottdiener JS, Bednarz J, Devereux R, et al. American Society of Echocardiography recommendations for use of echocardiography in clinical trials. J Am Soc Echocardiogr. 2004;17:1086-1119.
13. Inglese E, Leva L, Matheoud R, et al. Spatial and temporal heterogeneity of regional myocardial uptake in patients without heart disease under fasting conditions on repeated whole-body ${ }^{18}$ F-FDG PET/CT. J Nucl Med. 2007;48:16621669.

14. Choi Y, Brunken RC, Hawkins RA, et al. Factors affecting myocardial 2-[F-18]fluoro-2-deoxy-D-glucose uptake in positron emission tomography studies of normal humans. Eur J Nucl Med. 1993;20:308-318.

15. Fisher PW, Salloum F, Das A, Hyder H, Kukreja RC. Phosphodiesterase-5 inhibition with sildenafil attenuates cardiomyocyte apoptosis and left ventricular dysfunction in a chronic model of doxorubicin cardiotoxicity. Circulation. 2005;111:1601-1610.

16. Hrelia S, Fiorentini D, Maraldi T, et al. Doxorubicin induces early lipid peroxidation associated with changes in glucose transport in cultured cardiomyocytes. Biochim Biophys Acta. 2002;1567:150-156.

17. Carvalho RA, Sousa RP, Cadete VJ, et al. Metabolic remodeling associated with subchronic doxorubicin cardiomyopathy. Toxicology. 2010;270:92-98.

18. Tokarska-Schlattner M, Zaugg M, da Silva R, et al. Acute toxicity of doxorubicin on isolated perfused heart: response of kinases regulating energy supply. Am J Physiol Heart Circ Physiol. 2005;289:H37-H47.

19. Wakasugi S, Fischman AJ, Babich JW, et al. Myocardial substrate utilization and left ventricular function in Adriamycin cardiomyopathy. J Nucl Med. 1993;34:1529-1535.

20. Wang XY, Yang CT, Zheng DD, et al. Hydrogen sulfide protects H9c2 cells against doxorubicin-induced cardiotoxicity through inhibition of endoplasmic reticulum stress. Mol Cell Biochem. 2012;363:419-426.

21. Camici P, Marraccini P, Marzilli M, et al. Coronary hemodynamics and myocardial metabolism during and after pacing stress in normal humans. Am J Physiol. 1989;257:E309-E317.

22. Gilliam LA, Fisher-Wellman KH, Lin CT, Maples JM, Cathey BL, Neufer PD. The anticancer agent doxorubicin disrupts mitochondrial energy metabolism and redox balance in skeletal muscle. Free Radic Biol Med. 2013;65:988-996.

23. Lebrecht D, Setzer B, Ketelsen UP, Haberstroh J, Walker UA. Time-dependent and tissue-specific accumulation of mtDNA and respiratory chain defects in chronic doxorubicin cardiomyopathy. Circulation. 2003;108:2423-2429.

24. Tan-Chiu E, Yothers G, Romond E, et al. Assessment of cardiac dysfunction in a randomized trial comparing doxorubicin and cyclophosphamide followed by paclitaxel, with or without trastuzumab as adjuvant therapy in node-positive, human epidermal growth factor receptor 2-overexpressing breast cancer: NSABP B-31. J Clin Oncol. 2005;23:7811-7819. 